\title{
Identification of Forced Degradation Products of Lacosamide by LC-QqLIT-MS and LC-IT/TOF-MS
}

\author{
Na Zhou ${ }^{1 \dagger}$, Tao $\mathbf{L i}^{1,3 \dagger}$, Lianfeng $\mathrm{Ai}^{2}$, Chunhai Guo ${ }^{2}$, Juan Zhang ${ }^{1}$, Shan Fu ${ }^{1}$ and Qiao Wang ${ }^{1 *}$ \\ ${ }^{\prime}$ Department of Pharmaceutical Analysis, School of Pharmacy, Hebei Medical University, Shijiazhuang, 050017, P. R. China \\ Hebei Entry-Exit Inspection and Quarantine Bureau, Shijiazhuang, 050051, P. R. China \\ Department of Pharmacy, Hebei eye hospital, Xingtai, 054000, P. R. China
}

Received: 18 March 2017; accepted: 24 May 2017

\begin{abstract}
Lacosamide, a new type of antiepileptic drug, was subjected to forced degradation under the conditions of hydrolysis (acidic and alkaline), oxidation, dry heat, and photolysis to characterize its possible degradation products. The drug showed significant degradation under acidic, alkaline and oxidative conditions. The degradation products were separated on an Agilent Zorbax SB- $\mathrm{C}_{18}$ column with gradient elution using a mobile phase consisting of acetonitrile and ammonium acetate $(0.002 \mathrm{~mol} / \mathrm{L})$ with formic acid as additive. A combination of liquid chromatography hybrid triple quadrupole-linear ion trap mass spectrometry (LC-QqLIT-MS) and liquid chromatography hybrid ion trap/time-of-flight mass spectrometry (LC-IT/TOF-MS) was used to identify degradation products. A total of 7 products including 4 novel degradation products were characterized. The mechanisms of degradation products of lacosamide were discussed. Application of the method to study degradation products of lacosamide provided fragment information, allowing further investigation of the degradation pathways and intrinsic stability of the drug.
\end{abstract}

Keywords: lacosamide, forced degradation, LC-QqLIT-MS, LC-IT/TOF-MS, characterization

\section{Introduction}

Epilepsy is a clinical syndrome of chronic brain dysfunction that is characterized by unpredictable occurrence of seizures [1]. It is a common neurological disorder, and many antiepileptic drugs have been taken for seizure suppression [2, 3]. Lacosamide $((R)$-2-acetamido- $N$-benzyl-3-methoxypropionamide) is a new type of antiepileptic drug and approved by US and European regulatory authorities for use as add-on therapy for partial onset seizures in adults [4]. Compared to other antiepileptic drugs, lacosamide selectively enhances slow inactivation of voltage-gated sodium channels to achieve the purpose of seizure control $[5,6]$, which has a good effect on refractory partial epilepsy with less adverse reactions and drug interactions.

Degradation products formed during product development, transportation, and shelf life may have a significant impact on the quality and clinical safety of a drug. The existence of degradation products may also lead to the loss of drug activity and elicit possible adverse reactions. Therefore, forced degradation studies are recognized as an important part of drug development to identify the likely degradation products, which can in turn help establish the degradation pathways and intrinsic stability of the molecular and validate the stability-indicating power of the analytical procedures used [7-12].

A few high-performance liquid chromatography (HPLC) and liquid chromatography-mass spectrometry (LC-MS) methods for studying degradation products and impurities in

\footnotetext{
*Author for correspondence: Department of Pharmaceutical Analysis, Schoo of Pharmacy, Hebei Medical University, 361 East Zhongshan Road, Shijiazhuang 050017, P. R. China; Tel: +86-311-86265625; Fax: +86-311-86266419; E-mail address: qiaowang88@hotmail.com.

${ }^{\dagger} \mathrm{Na}$ Zhou and Tao Li contributed equally.
}

lacosamide have been reported [13-19]. Among them, three literature studies have been reported for the characterization of forced degradation products. All these studies used significantly different stress conditions, viz., acid and alkali stressor strength varied from 0.05 to $2 \mathrm{~mol} / \mathrm{L}$, temperature between $25^{\circ} \mathrm{C}$ and $80{ }^{\circ} \mathrm{C}$, and time from $15 \mathrm{~min}$ to $14 \mathrm{~h}$. A variable extent of degradation was observed. Tiwari et al. [13] identified and characterized 2 degradation products of lacosamide by using multi-stage $\left(\mathrm{MS}^{n}\right)$ and time-of-flight mass spectrometry (TOF-MS). Apexa et al. [14] characterized 3 degradation products of lacosamide. In addition, Ramisetti et al. [15] studied the degradation behavior of lacosamide under the International Conference on Harmonisation (ICH)-prescribed stress conditions and characterized 6 degradation products using electrospray-ionization quadrupole time-of-flight tandem mass spectrometry (ESI-Q-TOF$\mathrm{MS} / \mathrm{MS}$ ) technique. However, during our preliminary experiment, lacosamide underwent extensive degradation in acidic, alkaline, and oxidative degradation, which was different from what reported in the literature [15]. Moreover, there existed some novel products which have not been reported by any literature so far. Therefore, the purpose of this study were the following: (i) to conduct forced degradation study on lacosamide under the ICH Q1A(R2) prescribed conditions of hydrolysis, oxidation, dry heat, and photolysis to identify all possible degradation products; (ii) to characterize the degradation products by using liquid chromatography hybrid triple quadrupole linear ion trap mass spectrometry (LCQqLIT-MS) and liquid chromatography hybrid ion trap/timeof-flight mass spectrometry (LC-IT/TOF-MS); and (iii) to ascertain the degradation pathway of lacosamide, establish its inherent stability characteristics, and validate the feasibility of the analytical procedure used.

This is an open-access article distributed under the terms of the Creative Commons Attribution-NonCommercial 4.0 International License (https://creativecommons.org/licenses/by-nc/4.0/), which permits unrestricted use, distribution, and reproduction in any medium for non-commercial purposes, provided the original author and source are credited, a link to the CC License is provided, and changes - if any - are indicated. 


\section{Experimental}

Chemicals and Reagents. Lacosamide was synthesized by pharmaceutical chemistry lab of Hebei Medical University (Shijiazhuang, China). High-performance liquid chromatography (HPLC)-grade acetonitrile from J.T. Baker (Phillipsburg, NJ, USA) was used as the mobile phase. Formic acid and ammonium acetate were purchased from Dikma Technologies Incorporation. Analytical grade hydrochloric acid, sodium hydroxide, and $30 \% \mathrm{H}_{2} \mathrm{O}_{2}$ purchased from Tianjin Chemical Reagent Factory (Tianjin, China) were used to degrade lacosamide. Purified water was purchased from Wahaha Corporation (Hangzhou, China).

Analytical Instrumentation. LC-QqLIT-MS analysis was performed using a 3200 QTrap instrument (AB, Sciex, Framingham, MA, USA) coupled to an Agilent (Santa Clara, CA, USA) 1200 HPLC system. The HPLC system consists of an auto-sampler, a quaternary solvent delivery system and a column compartment. A hybrid triple quadrupole linear ion trap mass spectrometer equipped with Turbo $\mathrm{V}$ sources was used for detection. Analyst software (version 1.5.2, AB Sciex) was employed for instrument control and data processing.

LC-IT/TOF-MS experiments were conducted on a Shimadzu HPLC system (Kyoto, Japan) consisting of two LC-20AD pumps, an SIL-20AC auto-sampler, a CTO-20A column oven, an SPD-M20A PDA detector, and a CSM-20A system controller, which was coupled to an IT/TOF mass spectrometer (Shimadzu Corp., Kyoto, Japan) through an ESI interface.

Mass Spectrometry and Liquid Chromatography Conditions. The mass spectra of LC-QqLIT-MS were recorded both in the positive and negative electrospray ionization (ESI) mode. Nitrogen was used as nebulizer gas and curtain gas. The parameter settings of the ion source are as follows: curtain gas of $30 \mathrm{psi}$, an ion spray voltage of $5.5 /-4.5 \mathrm{kV}$, nebulizer gas of $60 \mathrm{psi}$, and heater gas of 65 psi. Enhanced mass spectrum-enhanced resolution spectrum-information-dependent acquisition-enhanced product ion (EMS-ER-IDA-EPI) scan mode was conducted on all of the degradation samples of lacosamide. Mass spectra were recorded in the range of $m / z$ 100-600 (MS) and $\mathrm{m} / \mathrm{z}$ 50-500 (MS/MS). The declustering potential (DP) was $35 \mathrm{~V} /-25 \mathrm{~V}$ and the collision energy (CE) was $\pm 25 \mathrm{eV}$ with a spread of $15 \mathrm{eV}$.

The parameter settings of IT/TOF-MS system are as follows: a full-scan MS with a mass range of $\mathrm{m} / \mathrm{z}$ 100-600, and data-dependent MS/MS acquisition on the suspected precursor ions were performed; the curved desolvation line (CDL) and heater block temperatures were both set at $200{ }^{\circ} \mathrm{C}$; nitrogen was used as nebulizer gas at a flow rate of $1.5 \mathrm{~L} / \mathrm{min}$; the MS/MS spectra were generated by using collision-induced dissociation (CID) of the selected precursor ions with a relative energy of $45 \%$. The ion accumulation time was set at $50 \mathrm{~ms}$. External mass calibration was performed prior to data acquisition using reference standard from $\mathrm{m} / \mathrm{z}$ 50 to $\mathrm{m} / \mathrm{z} 1000 \mathrm{Da}$, and all the calculated mass errors were less than 5 ppm.

Chromatographic separation was carried out on an Agilent Zorbax SB- ${ }_{18}$ column $(150 \mathrm{~mm} \times 4.6 \mathrm{~mm}, 5 \mu \mathrm{m})$ with gradient elution. The mobile phase consisted of solvent $\mathrm{A}$ ( $0.002 \mathrm{~mol} / \mathrm{L}$ ammonium acetate and $0.1 \%$ formic acid) and solvent B (acetonitrile). The gradient program was as follows: $88-70 \% \mathrm{~A}$ in $16 \mathrm{~min}, 70-55 \% \mathrm{~A}$ from $16 \mathrm{~min}$ to $20 \mathrm{~min}$, and $55 \%$ A from 20 to $25 \mathrm{~min}$. The column temperature was set at $30{ }^{\circ} \mathrm{C}$, and the injection volume was $5 \mu \mathrm{L}$. The total analysis time was $25 \mathrm{~min}$ with a pre-equilibration time of $8 \mathrm{~min}$. The same chromatographic conditions were used in the two applied MS systems.

Forced Degradation and Sample Preparation. The hydrolytic degradation studies on lacosamide were carried out in $1 \mathrm{~mol} / \mathrm{L} \mathrm{HCl}$ and $1 \mathrm{~mol} / \mathrm{L} \mathrm{NaOH}$ at $100{ }^{\circ} \mathrm{C}$ for $0.5 \mathrm{~h}$. Then, the degradation solutions of acidic and alkaline hydrolysis were neutralized with sodium hydroxide and hydrochloric acid, respectively. The oxidative degradation on lacosamide was carried out in $30 \%$ hydrogen peroxide $\left(\mathrm{H}_{2} \mathrm{O}_{2}\right)$ solution at $100{ }^{\circ} \mathrm{C}$ for $0.5 \mathrm{~h}$. The dry heat degradation was carried out by exposing the drug to a temperature of $105{ }^{\circ} \mathrm{C}$ for 14 days. Photolytic degradation study was carried out in a drug stability test chamber (Shanghai Linpin Instrument, China) by exposing the solid drug in the form of a thin layer in a Petri dish to $1.2 \times 10^{6}$ lux h of fluorescent and $200 \mathrm{Wh} / \mathrm{m}^{2} \mathrm{UV}$ light for 14 days. All the degradation samples were prepared at a concentration of $2 \mathrm{mg} / \mathrm{mL}$ in acetonitrile-ammonium acetate $(0.002 \mathrm{~mol} / \mathrm{L}, 0.1 \%$ formic acid) $(10: 90, v / v)$.

Analysis Process. Lacosamide and its degradation products were first analyzed by an LC-QqLIT-MS instrument. Probable structures were proposed by the comparison of mass fragmentation pathways of lacosamide and the degradation products. In order to increase the accuracy of the inference results, degradation products of lacosamide were then analyzed by LC-IT/TOF-MS, which provided accurate mass of both precursor and product ions.

Table 1. The designation, predicated elemental compositions, measured accurate masses, theoretical masses, mass errors, and major fragment ions of lacosamide and its degradation products

\begin{tabular}{|c|c|c|c|c|c|c|}
\hline Compounds & Designation & $\begin{array}{l}\text { Elemental } \\
\text { compositions } \\
\left([\mathrm{M}+\mathrm{H}]^{+}\right) \\
\end{array}$ & $\begin{array}{l}\text { Observed } \\
\text { mass }(m / z)\end{array}$ & $\begin{array}{l}\text { Theoretical } \\
\text { mass }(m / z)\end{array}$ & $\begin{array}{l}\text { Error } \\
(\mathrm{ppm})\end{array}$ & $\begin{array}{l}\text { Major product } \\
\text { ions }(\mathrm{m} / \mathrm{z})\end{array}$ \\
\hline 1 & Lacosamide & $\mathrm{C}_{13} \mathrm{H}_{19} \mathrm{~N}_{2} \mathrm{O}_{3}$ & 251.1385 & 251.1390 & -1.99 & $\begin{array}{l}\text { 219.1144, 177.1035, 144.0663, } \\
116.0726,108.0796,91.0545\end{array}$ \\
\hline 2 & 2-amino- $N$-benzyl-3-methoxypropanamide & $\mathrm{C}_{11} \mathrm{H}_{17} \mathrm{~N}_{2} \mathrm{O}_{2}$ & 209.1280 & 209.1285 & -2.39 & $\begin{array}{l}\text { 177.1019, 164. 1089, 148.0763, } \\
\text { 132.0820, 120.0823, 108.0835, } \\
91.0549\end{array}$ \\
\hline 3 & 2-acetamido- $N$-benzylacrylamide & $\mathrm{C}_{12} \mathrm{H}_{15} \mathrm{~N}_{2} \mathrm{O}_{2}$ & 219.1130 & 219.1115 & 6.84 & $\begin{array}{l}209.1265,144.0686,132.0880 \\
116.0726,108.0809,91.0549\end{array}$ \\
\hline 4 & $\begin{array}{l}\text { 2-acetamido- } N \text {-(2-hydroxybenzyl)- } \\
\text { 3-methoxypropanamide }\end{array}$ & $\mathrm{C}_{13} \mathrm{H}_{19} \mathrm{~N}_{2} \mathrm{O}_{4}$ & 267.1347 & 267.1339 & 2.99 & $\begin{array}{l}235.1074,161.0930,144.0664, \\
129.0672,116.0720,107.0489\end{array}$ \\
\hline 5 & $\begin{array}{l}\text { 2-acetamido- } N \text {-(4-hydroxybenzyl)- } \\
\text { 3-methoxypropanamide }\end{array}$ & $\mathrm{C}_{13} \mathrm{H}_{19} \mathrm{~N}_{2} \mathrm{O}_{4}$ & 267.1345 & 267.1339 & 2.24 & $\begin{array}{l}235.1015,144.0594,124.0775, \\
107.0488\end{array}$ \\
\hline 6 & $\begin{array}{l}\text { 2-acetamido- } N \text {-(hydroxy(phenyl)methyl)-3- } \\
\text { methoxypropanamide }\end{array}$ & $\mathrm{C}_{13} \mathrm{H}_{19} \mathrm{~N}_{2} \mathrm{O}_{4}$ & 267.1345 & 267.1339 & 2.24 & $\begin{array}{l}249.1234,217.0972,161.0921 \\
144.0655,124.0757,107.0500\end{array}$ \\
\hline 7 & 2-acetamido- $N$-benzyl-3-hydroxypropanamide & $\mathrm{C}_{12} \mathrm{H}_{17} \mathrm{~N}_{2} \mathrm{O}_{3}$ & 237.1231 & 237.1234 & -1.26 & $\begin{array}{l}\text { 193.0766, 171.1443, 108.0787, } \\
91.0594\end{array}$ \\
\hline 8 & $\mathrm{~N}$-benzyl-2-formamido-3-hydroxypropanamide & $\mathrm{C}_{11} \mathrm{H}_{15} \mathrm{~N}_{2} \mathrm{O}_{3}$ & 223.1086 & 223.1077 & 4.03 & $163.0874,149.0235,108.0814$ \\
\hline
\end{tabular}




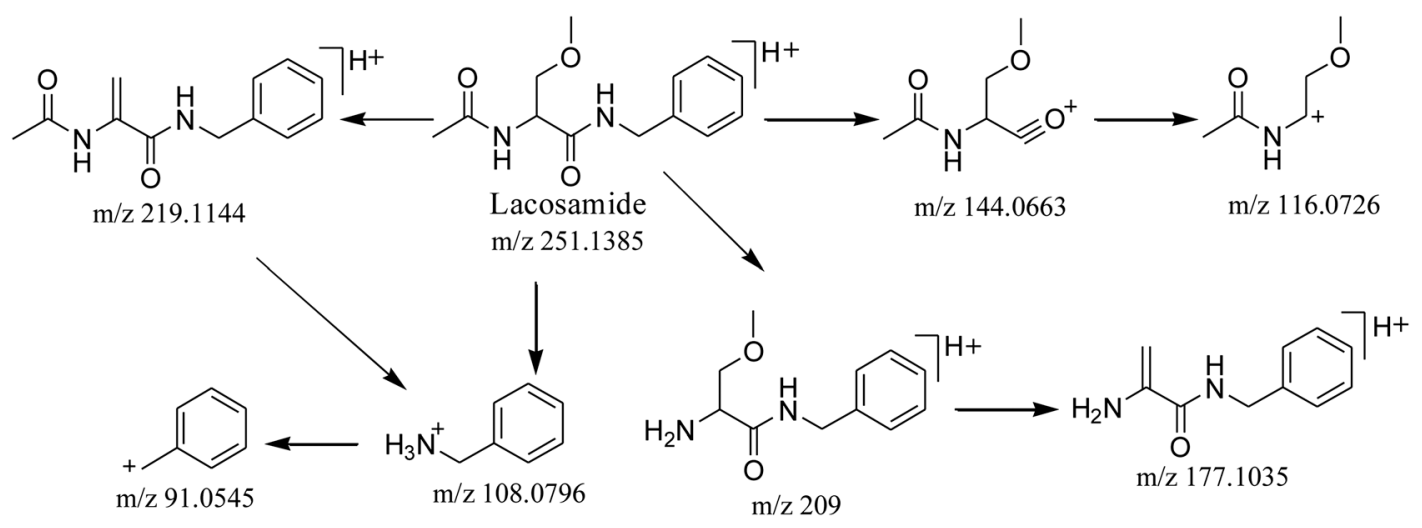

Figure 1. The fragmentation patterns of lacosamide obtained from the CID-MS/MS spectra

\section{Results and Discussion}

Fragmentation Patterns of Lacosamide. The MS data of lacosamide (compound $1, \mathrm{~m} / \mathrm{z}$ 251.1385) determined by IT/ TOF-MS was shown in Table 1 , and product ions at $\mathrm{m} / \mathrm{z}$ 219.1144, $\mathrm{m} / \mathrm{z} 177.1035, \mathrm{~m} / \mathrm{z} 144.0663, \mathrm{~m} / \mathrm{z} 116.0726, \mathrm{~m} / \mathrm{z}$ 108.0796, and $\mathrm{m} / \mathrm{z} 91.0545$ were observed. The fragmentation patterns of lacosamide are established and shown in Figure 1. The product ion at $\mathrm{m} / \mathrm{z} 219.1144$ was formed by the loss of methanol group from the parent ion. The elimination of acetyl group from the parent ion formed the fragment ion at $\mathrm{m} / \mathrm{z} 209$, which fragmented further to generate the ion at $\mathrm{m} / \mathrm{z} 177.1035$ through the loss of methanol group. Cleavage of the amide bond from the parent ion generate the peak at $\mathrm{m} / \mathrm{z} 144.0663$, which then produced the ion at $\mathrm{m} / \mathrm{z} 116.0726$ through the loss of $\mathrm{CO}$.

It should be noted that the product ion at $\mathrm{m} / \mathrm{z} 209$ emerged in the QqLIT mass spectrum was not detected in the IT/TOF mass spectrum of lacosamide (compound 1, $\mathrm{m} / \mathrm{z} 251.1385$ ). The reason for this may be related to the setting conditions and nature of the ion in the IT/TOF-MS environment.

Characterization of the Degradation Products. The LCQqLIT-MS total ion chromatograms (TICs) of the blank solvent and lacosamide degraded samples under acidic, alkaline, oxidative, dry heat, and photolytic stress conditions are presented in Figure 2 (A-F). A total of 7 degradation products, compounds $\mathbf{2 - 8}$, were identified and characterized. These degradation products were proposed based on the changes in $\mathrm{m} / \mathrm{z}$ values of precursor and product ions and those of precursor and lacosamide, and their elemental compositions obtained from LC-IT/TOF-MS are listed in Table 1. The table also shows the accurate masses of all the degradation products. MS/MS spectra of lacosamide and its degradation products obtained from IT/TOF-MS are listed in Figure 3. Structures of all the degradation products are shown in Figure 4.

Identification of Degradation Products from Prior Literature. Based on the mass spectra of the degradation products and the data reported in the literature [13-15], compounds $\mathbf{2}, \mathbf{3}$, and 7 were separately identified as 2-amino$\mathrm{N}$-benzyl-3-methoxypropanamide (compound 2, $\mathrm{m} / \mathrm{z} 209.1280$ ), 2-acetamido- $N$-benzylacrylamide (compound 3, $\mathrm{m} / \mathrm{z} 219.1130$ ), and 2-acetamido- $N$-benzyl-3-hydroxypropanamide (compound 7, $\mathrm{m} / \mathrm{z}$ 237.1231). The accurate masses of precursor and major product ions for these compounds determined by IT/TOF-MS are listed in Table 1.

Characterization of the Unknown Degradation Products. Under the oxidative condition, the extracted ion chromatogram (XIC) (Figure 2-E) of the ion at $\mathrm{m} / \mathrm{z} 267$ showed 3 peaks (Compounds 4, 5, and 6), and the mass spectra of them were similar. It was inferred that these 3 compounds were isomers, and the molecular mass of them were assigned as $266 \mathrm{Da}$ according to the ions at $\mathrm{m} / \mathrm{z} 267$ $\left([\mathrm{M}+\mathrm{H}]^{+}\right), m / z 289\left([\mathrm{M}+\mathrm{Na}]^{+}\right)$, and $m / z 305\left([\mathrm{M}+\mathrm{K}]^{+}\right)$ obtained from QqLIT-MS. The accurate masses of $[\mathrm{M}+\mathrm{H}]^{+}$of compounds $\mathbf{4}, \mathbf{5}$, and $\mathbf{6}$ calculated by IT/TOF-MS were $\mathrm{m} / \mathrm{z}$ $267.1347,267.1345$, and 267.1345 with errors of 2.99, 2.24, and $2.24 \mathrm{ppm}$, respectively. The nitrogen rule and elemental composition calculator suggested that the most likely molecular formula was $\left[\mathrm{C}_{13} \mathrm{H}_{19} \mathrm{~N}_{2} \mathrm{O}_{4}\right]^{+}$. The difference of $16 \mathrm{u}$ between the ion at $\mathrm{m} / \mathrm{z} 267$ and lacosamide at $\mathrm{m} / \mathrm{z} 251$ suggested that the compound might be a hydroxyl derivative of lacosamide. The presence of the ion at $\mathrm{m} / \mathrm{z} 107$ in compounds 4,5 , and $\mathbf{6}$, instead of the ion at $m / z 108$ in lacosamide, suggested that the benzyl amino cation was changed, and the most probable position of hydroxyl was benzylic carbon, $2-\mathrm{OH}$, and 4-OH of a benzene ring. For compound $\mathbf{6}$, the main product ions obtained from CID-MS/MS were at $\mathrm{m} / \mathrm{z}$ 249.1234, $\mathrm{m} / \mathrm{z}$ 217.0972, $\mathrm{m} / \mathrm{z}$ 161.0921, $\mathrm{m} / \mathrm{z} 144.0655, \mathrm{~m} / \mathrm{z} 124.0757$, and $\mathrm{m} / \mathrm{z}$ 107.0500. The product ion at $\mathrm{m} / z 249.1234$ was probably formed by the loss of $\mathrm{H}_{2} \mathrm{O}$, which suggested that the hydroxyl group existed in benzylic carbon. Therefore, compound $\mathbf{6}$ was identified as 2-acetamido- $N$-(hydroxy(phenyl)methyl)-3methoxypropanamide. For compounds $\mathbf{4}$ and $\mathbf{5}$, the calculated $C \log P$ values (Chemdraw software 7.0 ) were -0.32 and -0.27 , respectively. Based on the $C \log P$ data, the structure of compound 4 was tentatively worked out to be 2 -acetamido- $N$ (2-hydroxybenzyl)-3-methoxypropanamide, and the structure of compound $\mathbf{5}$ was identified as 2-acetamido- $N$-(4hydroxybenzyl)-3-methoxypropanamide. Besides, the calculated $C \log P$ of compound $\mathbf{6}$ was -0.0069 , so the retention time was longer than compounds $\mathbf{4}$ and $\mathbf{5}$, which is consistent with the identification of compound $\mathbf{6}$. The possible fragmentation pathways of compounds $\mathbf{4}, \mathbf{5}$, and $\mathbf{6}$ deduced from the CID-MS/MS analysis are shown in Figure 5.

Under the oxidative conditions, the ESI-MS spectrum of compound 8 measured by QqLIT revealed ions at $\mathrm{m} / \mathrm{z} 223$ $\left([\mathrm{M}+\mathrm{H}]^{+}\right), m / z 245\left([\mathrm{M}+\mathrm{Na}]^{+}\right)$in positive ion mode and $m / z 221\left([\mathrm{M}-\mathrm{H}]^{+}\right)$in the negative mode, from which the molecular mass of the compound was assigned as $222 \mathrm{Da}$. The accurate mass of $[\mathrm{M}+\mathrm{H}]^{+}$calculated by IT/TOF-MS was 223.1086 , with an error of $4.03 \mathrm{ppm}$. The elemental composition calculator suggested that the most likely molecular formula was $\left[\mathrm{C}_{11} \mathrm{H}_{15} \mathrm{~N}_{2} \mathrm{O}_{3}\right]^{+}$, which has a difference of $\mathrm{CH}_{2} \mathrm{CH}_{2}$ $(28 \mathrm{u})$ compared to lacosamide. The main product ions obtained from CID-MS/MS were at $\mathrm{m} / \mathrm{z}$ 163.0874, $\mathrm{m} / \mathrm{z}$ 149.0235, and $\mathrm{m} / \mathrm{z}$ 108.0814. The fragment ion at $\mathrm{m} / \mathrm{z}$ 163.0874 was formed by the loss of formamido and hydroxyl group. The ion at $\mathrm{m} / \mathrm{z} 149.0235$ was detected in the $\mathrm{MS}^{2}$ spectrum of $\mathbf{8}$ by the loss of methyl group. According to the oxidative stress conditions, the compound was tentatively identified 

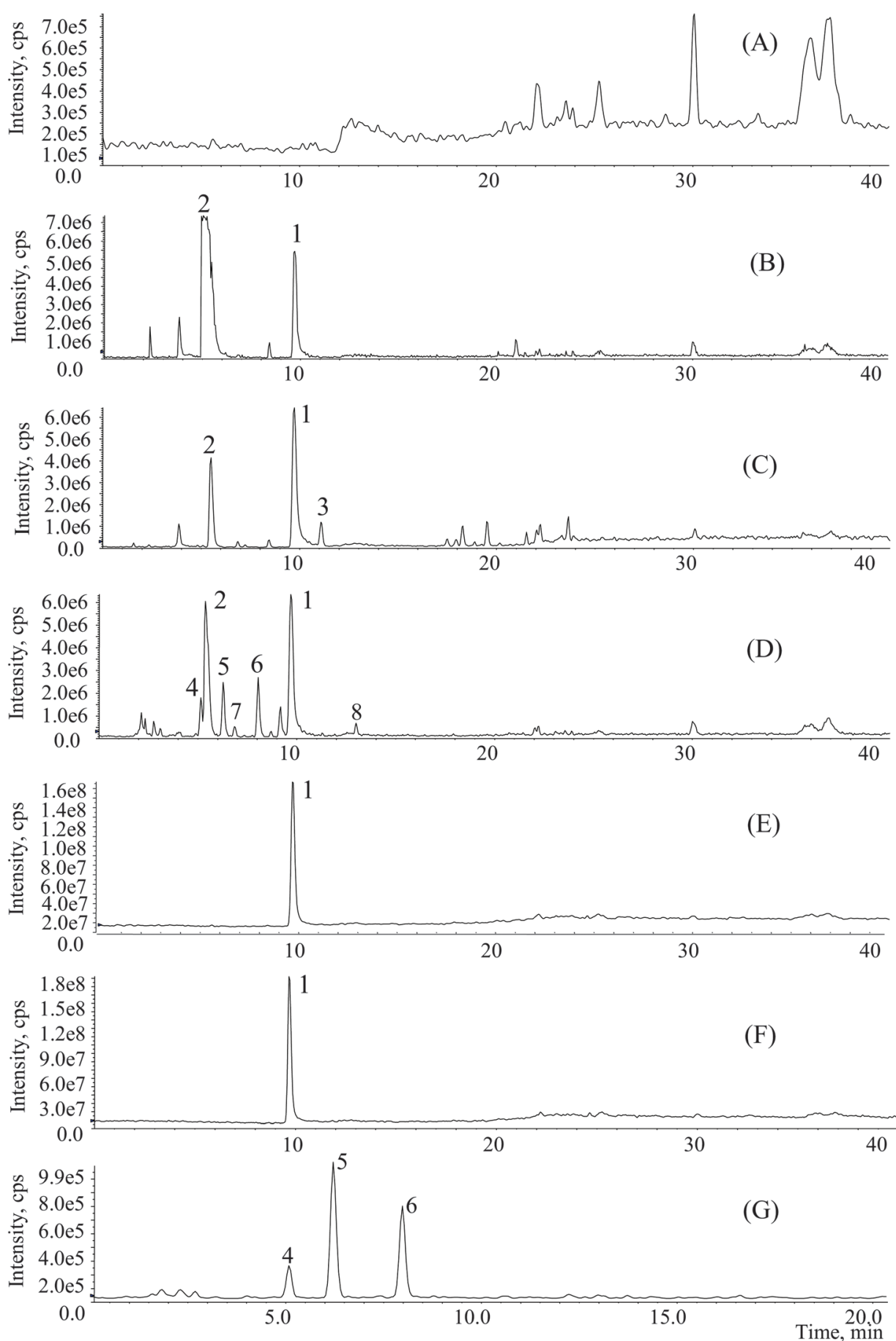

Figure 2. The LC-QqLIT-MS total ion chromatograms (TICs) of the degradation samples of lacosamide: (A) blank solvent, (B) acidic degradation, (C) alkaline degradation, (D) oxidative degradation, (E) dry heat degradation, and (F) photolytic degradation, and (G) the extracted ion chromatogram of the ion at $\mathrm{m} / \mathrm{z} 267$

as $\mathrm{N}$-benzyl-2-formamido-3-hydroxypropanamide. Structure of the compound was shown in Figure 4.

Degradation Behavior of Lacosamide. The percentage of peak area of the analyzed compounds under stressed conditions was shown in Table 2. As demonstrated in Table 2 and Figure 2, the drug primarily degraded into compound 2 which was formed under the acidic, alkaline, and oxidative stress conditions, while it was stable to dry heat and photolytic forced degradation. In comparison to acidic stress condition (Figure 2-B), one different degradation product, compound $\mathbf{3}$, was observed under the alkaline stress condition (Table 2, Figure 2-C). In this study, lacosamide showed an extensive degradation under the oxidative stress conditions
(Table 2, Figure 2-D), which are different from what is reported in the literature [15]. The results of this study suggest that lacosamide was unstable under the acidic, alkaline, and oxidative stress conditions, and should be stored in a tightly sealed container, protected from oxidizing agents.

In addition, some peaks in the total ion chromatograms of the degradation samples of lacosamide were still not identified due to the fewer fragment information, and degradation products with $\mathrm{m} / \mathrm{z}$ values at $\mathrm{m} / \mathrm{z} 108, \mathrm{~m} / \mathrm{z} 177$, and $\mathrm{m} / \mathrm{z} 251$ reported by Ramisetti et al. [15] were not observed in this study. The possible reason may be due to employment of less duration of stressing in our case, the minor amounts of the degradation products, and there is a little time for the 


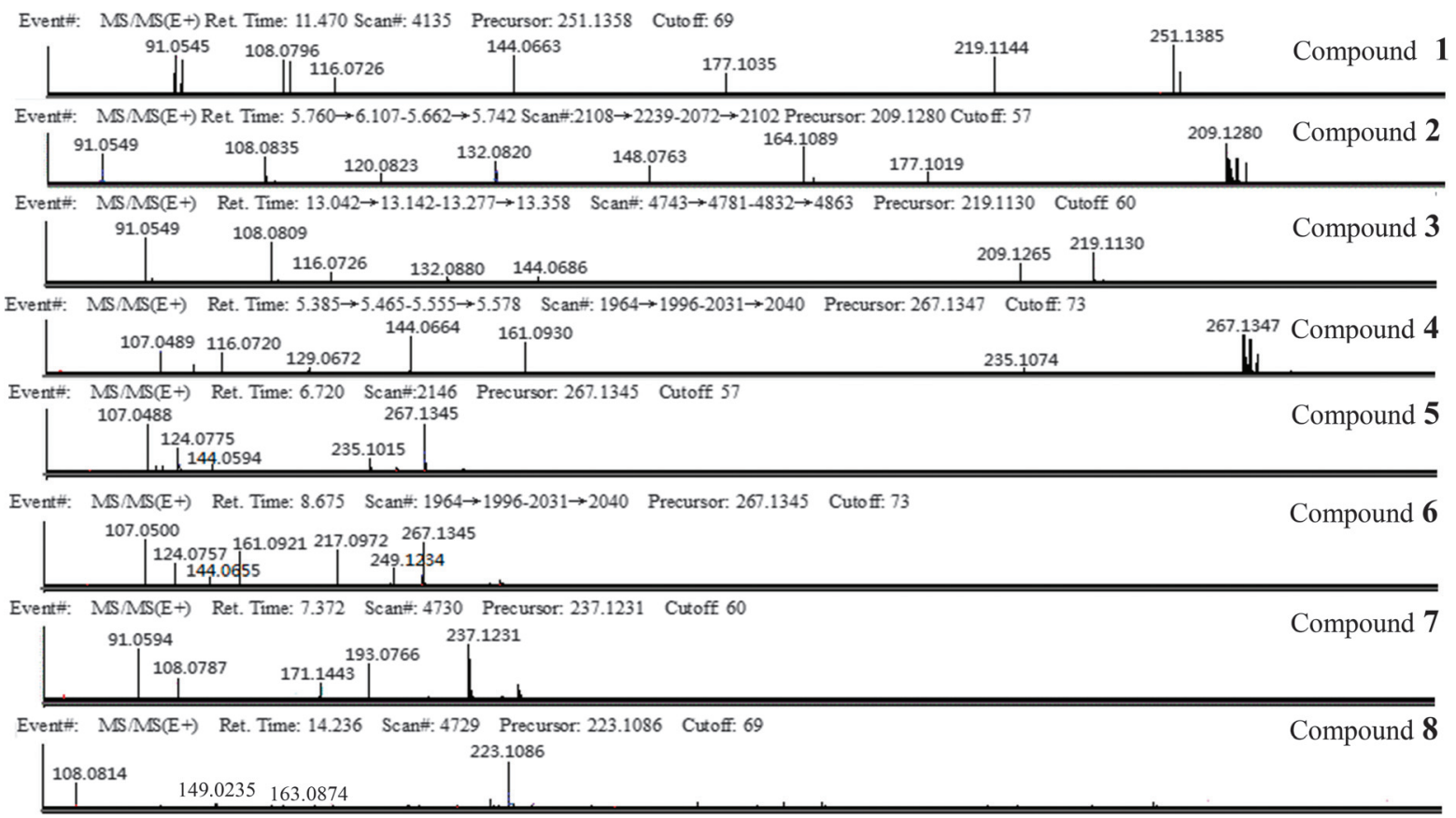

Figure 3. Mass spectra of lacosamide and its degradation products 2-8

fragments to be trapped as the degradation products elute from the LC column. Besides, IT/TOF-MS belongs to multi-stage ion trap tandem mass spectrometry, the instability of which would also affect the monitoring results.

Degradation Pathway. The most probable pathway explanations including oxidative, acidic, and alkaline hydrolytic pathway for formation of impurities 2-8 from lacosamide were outlined in Figure 4. (a) In oxidative condition, there were 3 main kinds of reaction: one was the breakage of amide bond, one was proposed to be peroxide-assisted hydroxylation, and another was the fracture of ether bond. (b) In acidic hydrolytic conditions, there was only one type of reaction, the breakage of amide bond. (c) Under alkaline hydrolytic conditions, there were two classes of reaction, one was the breakage of amide bond, and the other was the fracture of ether bond.<smiles>COCC(N)C(=O)NCc1ccccc1</smiles>

compound 2 $\mathrm{m} / \mathrm{z} 209$<smiles>C=C(NC(C)=O)C(=O)NCc1ccccc1</smiles>

compound 3 $\mathrm{m} / \mathrm{z} 219$<smiles>CC(=O)NC(CO)C(=O)NCc1ccccc1</smiles>

compound 7

$\mathrm{m} / \mathrm{z} 237$<smiles>O=COc1ccccc1</smiles><smiles>CC(C)O</smiles><smiles>COCC(NC(C)=O)C(=O)NCc1cccc(CCCCCCCCCCC(=O)NC(COC)C(=O)NCc2ccc(O)cc2)c1O</smiles>
compound 1 $\mathrm{m} / \mathrm{z} 267$<smiles>COCC(NC(C)=O)C(=O)NC(O)c1ccccc1</smiles>

Figure 4. Structures of the degradation products of lacosamide 


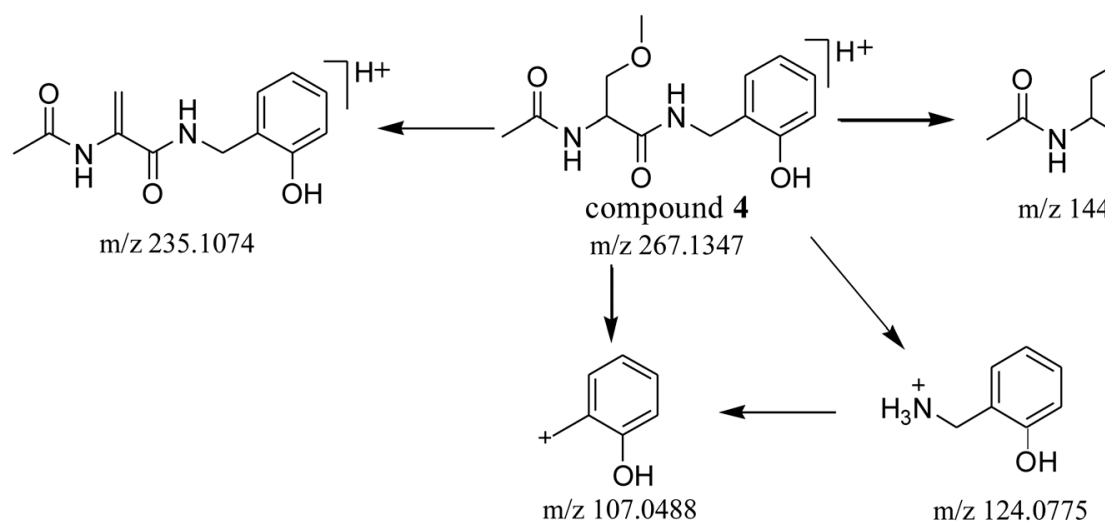

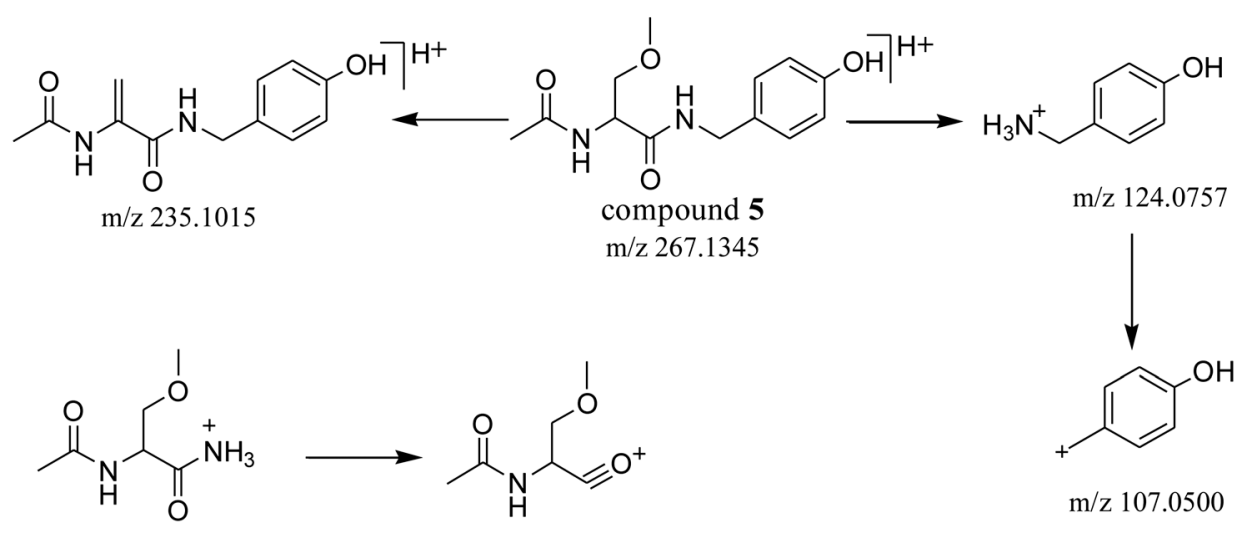

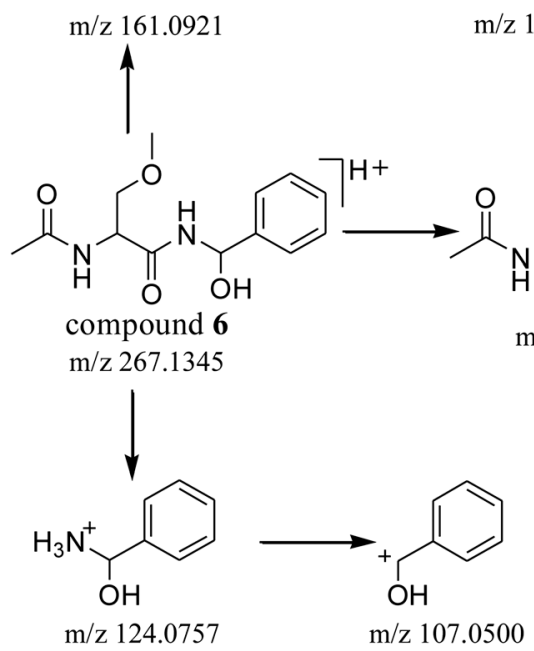<smiles>[NH3+]Cc1ccccc1</smiles>

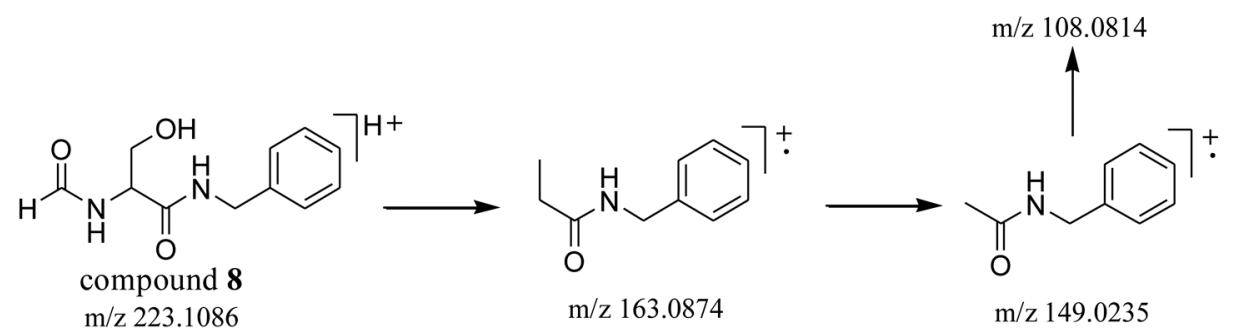

Figure 5. The fragmentation pathways of compounds 4, 5, 6, and 8

Table 2. The percentage of peak area of the analyzed compounds under stressed conditions

\begin{tabular}{llllll}
\hline Compounds & Acidic degradation & Alkaline degradation & Oxidation degradation & Dry heat degradation & Hot degradation \\
\hline $\mathbf{1}$ & $9.3 \%$ & $79.2 \%$ & $53.1 \%$ & $100 \%$ & $100 \%$ \\
$\mathbf{2}$ & $90.7 \%$ & $19.3 \%$ & $23.1 \%$ & - & - \\
$\mathbf{3}$ & - & $1.5 \%$ & - & - & - \\
$\mathbf{4}$ & - & - & $4.9 \%$ & - & - \\
$\mathbf{5}$ & - & - & $7.5 \%$ & - & - \\
$\mathbf{6}$ & - & - & $10.4 \%$ & - & - \\
$\mathbf{7}$ & - & - & $0.7 \%$ & - & - \\
$\mathbf{8}$ & - & & & & \\
& & & & &
\end{tabular}




\section{Conclusions}

LC-IT/TOF-MS was used to characterize the degradation products of lacosamide under acidic, alkaline, oxidative, dry heat, and photolytic stress conditions and simultaneously verify the results of LC-QqLIT-MS. A total of 7 degradation products were analyzed and 4 degradation products under the oxidative conditions were detected and identified for the first time. Most probable structures of the degradation products were assigned, and the accurate masses were calculated with errors between the observed and theoretical masses within $7 \mathrm{ppm}$. The results in the present study indicated that lacosamide was unstable under the acidic, alkaline, and oxidative stress conditions and the most susceptible positions for degradation in the molecule were the amido bond, ether bond, benzylic carbon, and benzene ring. The plausible degradation pathway for formation of the degradation products was laid out.

Acknowledgements. This work was supported by the National Natural Science Foundation of China (81102412), the Ministry of Education Key Project of Science and Technology Foundation of China (211021), Hundreds of Innovative Talents Project of Hebei Education Department of China, and the Natural Science Foundation of Hebei Province of China (C2011206158, 08B031).

\section{References}

1. Wang, X. F. Int. J. Intern. Med. 1988, 11, 511-513.

2. Jin, Z. B.; Sun, W. H. Chin. J. New Drugs 2005, 5, 544-547.

3. Spanaki, M. V.; Barkley, G. L. Neurology: Clin. Pract. 2012, 9 , 236-241.

4. Chung, S.; Sperling, M. R.; Biton, V., et al. Epilepsia 2010, 1, 27.

5. Harris, J. A.; Murphy, J. A. Ann. Pharmacother 2009, 43, 1809-1817.

6. Halford, J. J.; Lapointe, M. Epilepsy Curr. 2009, 9, 1-9.

7. Liu, Z. Y.; Zhang, H. H.; Chen, X. H.; Zhou, X. N.; Wan, L.; Sun, Z. L. Int. J. Mass Spectrom. 2011, 303, 90-96.

8. Namdev, D.; Borkar, R. M.; Raju, B.; Kalariya, P. D.; Rahangdale, V. T.; Gananadhamu, S.; Srinivas, R. J. Pharm. Biomed. Anal. 2014, 88, 245-255. $6-17$.

9. Handa, T.; Singh, S.; Singh, I. P. J. Pharm. Biomed. Anal. 2014, 89,

10. Rao, R. N.; Ramachandra, B.; Sravan, B.; Khalid, S. J. Pharm. Biomed. Anal. 2014, 89, 28-33.

11. Ramesh, T.; Rao, P. N.; Rao, R. N. J. Pharm. Biomed. Anal. 2014, 88 , 609-616.

12. ICH, Stability Testing of New Drug Substance and Products Q1A(R2), in: International Conference on Harmonization, IFPMA, Geneva, 2003.

13. Tiwari, R. N.; Bonde, C. G. J. Liq. Chromatogr. $R T$ 2014, 37, 2046-2061.

14. Patel, A.; Suhagia, B. N.; Patwari, A. Int. J. Pharm. Pharm. Sci. 2013 6, 593-599.

15. Ramisetti, N. R.; Kuntamukkala, R.; Lakshetti, S.; Sripadi, P. J. Pharm. Biomed. Anal. 2014, 95, 256-264.

16. Molleti, S.; Rao, V.; Jayaveera, K. N. Pharma. Chem. 2013, 5, 81-89.

17. Parmar, M. D.; Nimavat, K. S.; Vyas, K. B.; Rao, D. V. N. S.; Pande, R. Int. J. Pharm. Res. Scholars 2012, 1, 40-47.

18. Chakravarthy, V. K.; Sankar, D. G. Rasayan J. Chem. 2012, 5, 293-310.

19. Chakravarthy, V. K.; Shankar, D. G. Rasayan J. Chem. 2011, 4, 